\title{
BEITRÄGE ZUR KOLORIMETRISCHEN HUMUSBESTIMMUNG IM BODEN
}

\author{
EUGEN BARKOFF
}

\begin{abstract}
Landwirtschaftliche Versuchsanstalt, Abteilung für Agrikulturchemie und-physik, Tikkurila
\end{abstract}

Eingegangen am 22.10. 1954

Für die Humusbestimmung durch nasse Verbrennung mittels Chromschwefelsäure sind in der agrikulturchemischen und bodenkundlichen Literatur viele verschiedene Methoden vorgeschlagen worden. Sie gründen sich alle darauf, dass der Humuskohlenstoff zu Kohlendioxyd oxydiert wird, während das sechswertige Chrom gleichzeitig nach der folgenden Formel zu dreiwertigem reduziert wird:

$$
2 \mathrm{Cr}_{2} \mathrm{O}_{7}^{--}+16 \mathrm{H}^{+}+3 \mathrm{C} \rightarrow 4 \mathrm{Cr}^{+++}+8 \mathrm{H}_{2} \mathrm{O}+3 \mathrm{CO}_{2} \text {. }
$$

Aus dieser Formel geht hervor, dass 2 Moleküle oder 12 Äqu Dichromat 3 Kohlenstoffatome und demnach $1 \mathrm{mg}$-Äqu Dichromat $3 \mathrm{mg}$ Kohlenstoff oxydieren können. Der Humus enthält durchschnittlich $58 \%$ Kohlenstoff und 1 mig Kohlenstoff entspricht somit $1.724 \mathrm{mg}$ Humus, so dass $1 \mathrm{mg}$ - ̈̈qu Dichromat $5.172 \mathrm{mg}$ Humus oxydieren kann.

Anfangs hat man die bei der Oxydation unverbrauchte Dichromatmenge entweder durch die jodometrische oder ferrometrische Titration bestimmt und daraus die dem Humusgehalt entsprechende Menge des verbrauchten Dichromats berechnet. In der letzten Zeit haben dagegen viele Forscher die bei der Oxydation gebildete Chromimenge gemessen, was kolorimetrisch leicht durchführbar ist.

Die Tabelle 1 zeigt, in welcher Beziehung sich die von verschiedenen Forschern angewendeten Methoden, den Humus in der Chromschwefelsäure zu bestimmen, voneinander unterscheiden. Daraus ersieht man, dass zur Analyse Bodenmengen verschiedener Grösse, 0,5-10 g, je nach dem Humusgehalt, gebraucht worden sind. Als Oxydationsmittel hat man verschiedene Mengen 1 oder 2 n Kaliumdichromatlösung benutzt.

Konzentrierte Schwefelsäure hat man entweder direkt dem Boden, 5-40 ml, oder erst nach Zufügung der Dichromatlösung, 10-26 ml, zugesetzt. Die weitere Behandlung ist alternativ folgendermassen ausgeführt worden: 


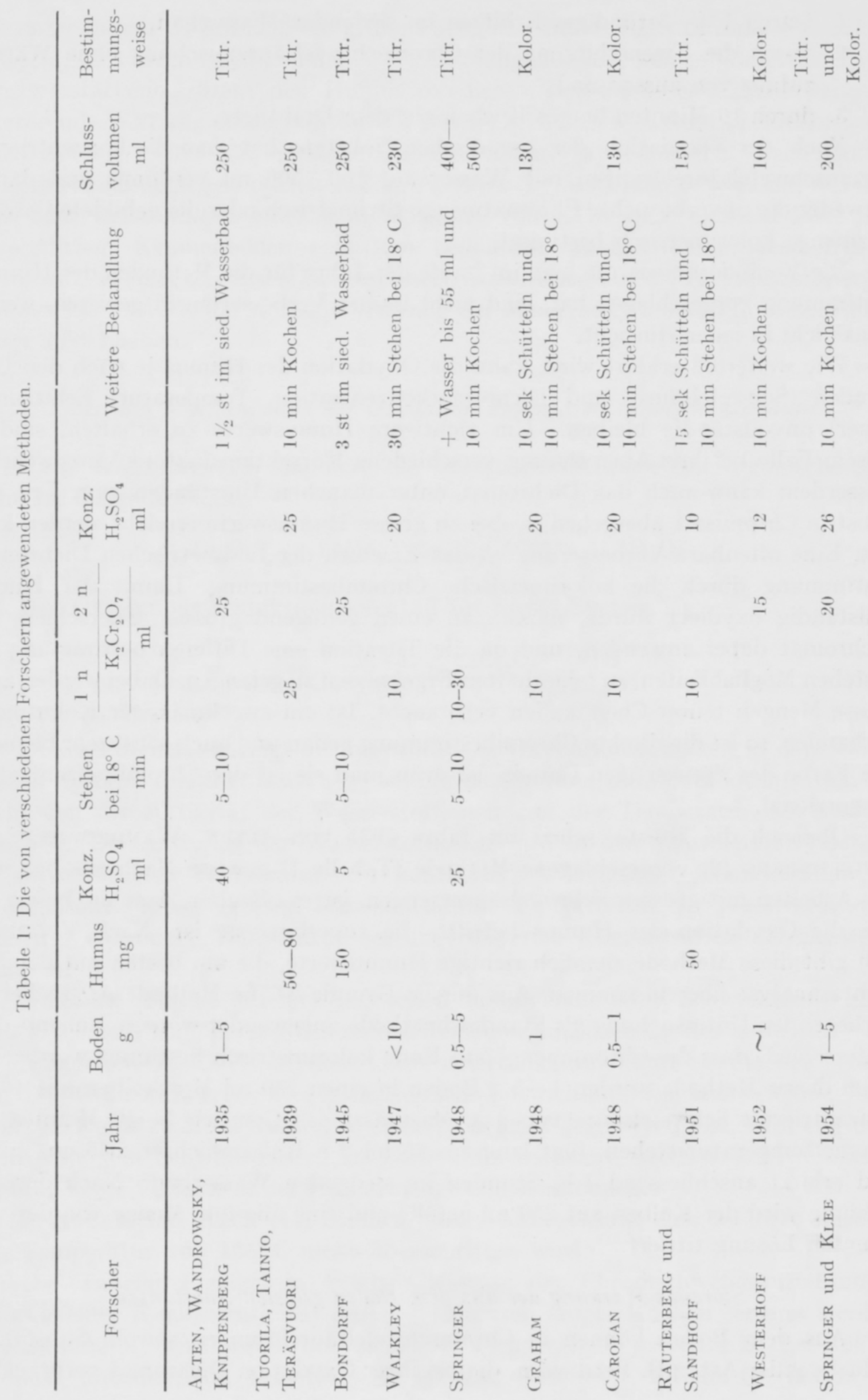


1. Durch $1 \frac{1}{2}-3$ stündiges Erhitzen im siedenden Wasserbad,

2. durch die Eigenerhitzung der Chromschwefelsäuremischung ohne Wärmezufuhr von aussen und

3. durch 10 Minuten langes Kochen auf dem Drahtnetz.

Nach der Oxydation der organischen Substanz hat man die konzentrierten Chromschwefelsäurelösungen mit Wasser auf $100-500 \mathrm{ml}$ verdünnt und darauf entweder die unverbrauchte Chromatmenge titrimetrisch oder die gebildete Chromisalzmenge kolorimetrisch bestimmt.

Die Veränderungen, die man im Laufe der Jahre für die Methoden der Humusbestimmung vorgeschlagen hat, sind nicht immer Verbesserungen gewesen, wenigstens nicht in jeder Hinsicht.

Wie weiterhin erklärt wird, kann die Oxydation des Humus je nach den Umständen (Schwefelsäure- und Dichromatkonzentration, Temperatur, Erhitzungsdauer) unvollständig bleiben. Um richtigere Humuswerte zu erhalten, sind in diesem Falle bei ihrer Ausrechnung verschiedene Korrektionsfaktoren anzuwenden. Ausserdem kann auch das Dichromat unter manchen Umständen zum Teil von selbst in Chromisalz übergehen, wobei zu grosse Humuswerte erreicht werden können. Eine offenbare Verbesserung ist das Ersetzen der titrimetrischen Dichromatbestimmung durch die kolorimetrische Chromibestimmung. Damit der Humus vollständig oxydiert würde, muss man einen genügend grossen Überschuss von Dichromat dabei anwenden, und da die Titration eine Differenzbestimmung ist, bestehen Möglichkeiten, zu fehlerhaften Ergebnissen zu gelangen. Dabei werden auch grosse Mengen teurer Chemikalien verbraucht. Ist ein zweckmässiger Kolorimeter vorhanden, so ist die direkte Chromibestimmung genau und auch sonst sehr bequem. Die Farbe des dreiwertigen Chroms ist grün, und sie ist der Chromikonzentration proportional.

Obgleich die älteste, schon im Jahre 1935 von Alten, Wandrowsky und KnippenberG (2) vorgeschlagene Methode (Tabelle 1) gewisse Nachteile hat, u.a. das Arbeiten mit grossen Schwefelsäuremengen, ist es offenbar, dass sie, besonders was die Oxydation des Humus betrifft, die zuverlässigste ist. Nach VuORINEN (13) gibt diese Methode ziemlich richtige Humuswerte, die am besten mit der Elementaranalyse übereinstimmen. Aus diesem Grunde ist die Methode Alten bei der vorliegenden Untersuchung als Standardmethode angewendet worden, nur mit dem Unterschied, dass die Chromimenge am Ende kolorimetrisch bestimmt worden ist. Nach dieser Methode werden 1-5 g Boden in einen $250 \mathrm{ml}$ Masskolben mit $40 \mathrm{ml}$ konzentrierter Schwefelsäure versetzt. Man lässt das Gemisch 5-10 Minuten bei Zimmertemperatur stehen, fügt langsam $25 \mathrm{ml} 2 \mathrm{n}$ Kaliumdichromatlösung hinzu und erhitzt anschliessend $1 \frac{1}{2}$ Stunden im siedenden Wasserbad. Nach der Abkühlung wird der Kolben auf $250 \mathrm{ml}$ gefüllt und eine aliquote Menge von der verdünnten Lösung titriert.

\section{Störende Wirkung der aus dem Boden gelösten Substanzen}

Aus dem Boden können in Chromschwefelsäurelösungen sowohl Substanzen reduzierender Art, z.B. Ferroeisen, die bei ihrer Oxydation Dichromat verbrauchen 
und unter diesen Umständen die grüne Farbe des dreiwertigen Chroms verstärken, als auch Substanzen oxydierender Art, z.B. Manganoxyde, welche, die grüne Farbe nicht verstärkend, direkt den Humus oxydieren, sich lösen. Glücklicherweise ist die störende Wirkung dieser Substanzen gering. Z.B. 1 Gewichtsteil Ferroeisen kann gleich viel wie 0.0926 Gewichtsteile Humus das Chromat reduzieren. Nach Aarnio (1) enthielt ein an Eisen reichster Ton, $\mathrm{A}_{2}$-Horizont, ungefähr $3 \%$ Eisen, das sich nach dem vielstũndigen Kochen in Salzsäure von 1.115 spez. Gewicht löste. Die gewöhnlichen Krumenböden enthalten bedeutend weniger Eisen, besonders in Ferroform, so dass das Eisen keine grössere Einwirkung auf die Humusbestimmung ausüben kann. Auch die störende Wirkung der Manganoxyde kann in diesem Falle ausser acht bleiben.

\section{Über den Mechanismus der Oxydation durch die Chromschwefelsäure}

Nach Walkley (14) oxydiert die Chromschwefelsäure eine organische Substanz auf folgende Weise:

$$
\mathrm{Cr}_{2} \mathrm{O}_{7}^{--}+14 \mathrm{H}^{+}+6 \mathrm{e}^{-} \rightleftarrows 2 \mathrm{Cr}^{+++}+7 \mathrm{H}_{2} \mathrm{O} .
$$

Das Oxydations-Reduktionspotential für dieses System ist:

$$
\mathrm{E}=1.36+\frac{\mathrm{R} \cdot \mathrm{T}}{\mathrm{n} \cdot \mathrm{F}} \log \frac{\left[\mathrm{Cr}_{2} \mathrm{O}_{7}^{--}\right] \cdot\left[\mathrm{H}^{+}\right]^{14}}{\left[\mathrm{Cr}^{+++}\right]^{2}} .
$$

Dieses Potential E zeigt die Intensität der Oxydationsfähigkeit in dem vorliegenden System, und ihre Stärke ist bei der bestimmten Chromatkonzentration vor allem von der Aktivität der Wasserstoffionen und der Temperatur des Systems abhängig. Die Aktivität der Wasserstoffionen wird in den Schwefelsäurelösungen bei unveränderter Temperatur mit kontinuierlich steigender Konzentration der Schwefelsäure immer grösser. Daneben nimmt die Aktivität der Wasserstoffionen auch mit steigender Temperatur bei unveränderter Konzentration der Schwefelsäure zu.

SAlONEN (9) hat gezeigt, dass schon die ungefähr $60 \%$ Schwefelsäure allein bei $120^{\circ} \mathrm{C}$ die Verminderung des reduzierenden organischen Stoffes bewirkte.

Auch nach OkNin (7) oxydiert konzentrierte Schwefelsäure bei der Temperatur von $100^{\circ} \mathrm{C}$ an aufwärts unter gleichzeitiger Entwicklung von Schwefeldioxydgas amorphe Kohle zu Kohlensäure. Aber falls Chromat gegenwärtig ist, wird es gleichzeitig von dem Schwefeldioxyd zu Chromisalz reduziert, so dass sich wenigstens dadurch keine Verluste in der Humusmenge ergeben können.

SPRINGer und KleE (11) machen darauf aufmerksam, dass bei ihrer Methode eine Temperatur von $165^{\circ} \mathrm{C}$ nicht überschritten wird.

Die Tabelle 2 zeigt, in welchen Mengen die Chromschwefelsäurelösungen verschiedener Konzentrationen durch 15 Minuten langes Kochen zersetzt werden. Die Lösung 1 wird auch bei niedriger Temperatur, jedoch z.B. bei $120^{\circ} \mathrm{C}$ ziemlich unbedeutend, zersetzt. Es ist also vorteilhaft, für die Oxydation des 
Tabelle 2. Zersetzung der Chromschwefelsäurelösungen durch 15 min langes Kochen.

\begin{tabular}{|c|c|c|c|c|c|c|}
\hline \multirow{3}{*}{ Lösung } & \multicolumn{3}{|c|}{ Herstellung der Lösungen } & \multirow{3}{*}{$\begin{array}{c}\text { Wasser auf } \\
10 \mathrm{ml} 96 \%_{\mathrm{H}_{2} \mathrm{SO}_{4}} \mathrm{ml}^{2}\end{array}$} & \multirow{3}{*}{$\begin{array}{c}\text { Siedetemp. } \\
\text { in }{ }^{\circ} \mathrm{C} \\
\text { etwa }\end{array}$} & \multirow{3}{*}{$\begin{array}{c}\mathrm{mg}-\mathrm{Aqu} \\
\mathrm{Cr}^{+++}\end{array}$} \\
\hline & $10 \mathrm{n} \mathrm{CrO}_{3}$ & Wasser & $96 \% \mathrm{H}_{2} \mathrm{SO}_{4}$ & & & \\
\hline & 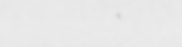 & $\mathrm{ml}$ & & & & \\
\hline 1 & 5 & 0 & 10 & 4.46 & 198 & 44 \\
\hline 2 & 5 & 1 & 10 & 5.46 & 181 & 24 \\
\hline 3 & 5 & 2 & 10 & 6.46 & 174 & 9 \\
\hline 4 & 5 & 3 & 10 & 7.46 & 162 & 3 \\
\hline 5 & 5 & 4 & 10 & 8.46 & 158 & 1 \\
\hline 6 & 5 & 5 & 10 & 9.46 & 150 & $<1$ \\
\hline
\end{tabular}

Humus möglichst konzentrierte Chromschwefelsäurelösungen bei einer genügend hohen Temperatur anzuwenden, wenn auch nicht zu hohe, damit sich das Chromat nicht von selbst zersetzt.

Wie die Tabelle 1 zeigt, führen einige Forscher die Oxydationsreaktion in den Chromschwefelsäurelösungen ohne Wärmezufuhr von aussen aus, sondern benutzen für die Eigenerhitzung nur die Lösungswärme der konzentrierten Schwefelsäure und die Verbrennungswärme des Humus. Welche Temperatur die Lösung dabei erreichen kann, ist hauptsächlich sowohl von der Proportion der verbrauchten Wasser- und Schwefelsäuremengen als auch von der Wärmemenge abhängig, die dieses Gemisch an die Umgebung verliert.

In der Figur 1 ist dargestellt (Kurvenpaar I), welche Temperatur das Gemisch durch Hinzufügen verschieden grosser Wassermengen zu $96 \%$ und $100 \%$ Schwefelsäure erhalten kann, wenn die ganze Reaktionswärme ausschliesslich für die Erhitzung des Gemisches verbraucht würde. Die Werte für dieses Kurvenpaar wurden durch die aus den physikalisch-chemischen Tabellen (LANDOLT-BöRnsteIn) erhaltenen molekularen Lösungswärmen flüssiger Schwefelsäure und die spezifischen Wärmen wässeriger Schwefelsäurelösungen berechnet. Wie ersichtlich, ergibt sich die höchste Temperatur, $152^{\circ} \mathrm{C}$, wenn man nach dem Volumen 10 Teile $96 \%$ Schwefelsäure und 5 Teile Wasser miteinander mischt. Bei mehr konzentrierten Schwefelsäuren kann eine noch höhere Temperatur, z.B. $181^{\circ} \mathrm{C}$ bei $100 \%$ Schwefelsäure, erreicht werden. Praktisch erhält man jedoch eine bedeutend niedrigere Temperatur. Alle diese Umstände sind bei der Humusbestimmung ohne Wärmezufuhr von aussen in Betracht zu ziehen.

Das Kurvenpaar II zeigt die Siedetemperatur von Schwefelsäurelösungen verschiedener Konzentrationen. Demnach siedet eine Mischung, die ein Teil 96\% Schwefelsäure und ein Teil Wasser enthält, bei $146^{\circ} \mathrm{C}$ und ihre Siedetemperatur steigt über $165^{\circ} \mathrm{C}$ erst dann, nachdem ein Viertel von der ganzen Wassermenge der Mischung verdunstet ist, wobei die Zersetzung des Chromats schon bemerkbar wird. Dies ist wieder, um Extrazersetzung des Chromats zu vermeiden, bei der Oxydation des Humus durch Kochen in Betracht zu ziehen. 
Das Kurvenpaar III zeigt die Konzentrationen der Schwefelsäurelösungen in Gewichtsprozent.

Wenn man für eine Analyse je nach dem Gehalt des Humus eine bestimmte Bodenmenge nehmen soll und dazu eine genügend grosse Chromatmenge braucht, so ist die erforderliche Schwefelsäuremenge von derjenigen Wassermenge, die mit der Chromatlösung dem Boden zugesetzt ist, abhängig. Mit kleineren Schwefelsäuremengen kann man dabei nur durch Anwenden von leichter löslichen Chromatquellen als Kaliumdichromat auskommen. Eine solche Substanz ist Chromtrioxyd, das etwa zehnmal mehr als Kaliumdichromat löslich und dazu noch ein Säureanhydrid ist.

Das rote Chromtrioxyd wird als ein stark polymerisiertes Produkt $\left(\mathrm{CrO}_{3}\right) \mathrm{x}$, welches im Wasser jedoch schnell depolymerisiert wird, betrachtet. Verdünnte Wasserlösungen enthalten vorzugsweise $\mathrm{H}_{2} \mathrm{Cr}_{2} \mathrm{O}_{7}$ und $\mathrm{H}_{2} \mathrm{CrO}_{4}$, die sich folgendermassen dissoziieren:

$$
\begin{aligned}
& \mathrm{H}_{2} \mathrm{CrO}_{4} \rightleftarrows \mathrm{H}^{+}+\mathrm{HCrO}_{4}^{-} \\
& \mathrm{HCrO}_{4} \rightleftarrows \mathrm{H}^{+}+\mathrm{CrO}_{4}^{--} \\
& \mathrm{H}_{2} \mathrm{Cr}_{2} \mathrm{O}_{7} \rightleftarrows \mathrm{H}^{+}+\mathrm{HCr}_{2} \mathrm{O}_{7}^{-} \\
& \mathrm{HCr}_{2} \mathrm{O}_{7}^{-} \rightleftarrows \mathrm{H}^{+}+\mathrm{Cr}_{2} \mathrm{O}_{7}^{--} .
\end{aligned}
$$

Zwischen diesen besteht folgendes Gleichgewicht:

$$
2 \mathrm{HCrO}_{4}^{-} \rightleftarrows \mathrm{Cr}_{2} \mathrm{O}_{7}^{--}+\mathrm{H}_{2} \mathrm{O} \text {. }
$$

In den mehr konzentrierten Chromsäurelösungen bilden sich durch die Polymerisation die Trichromat- und Tetrachromationen

$$
\begin{gathered}
\mathrm{HCrO}_{4}^{-}+\mathrm{HCr}_{2} \mathrm{O}_{7}^{-} \rightleftarrows \mathrm{Cr}_{3} \mathrm{O}_{10}{ }^{--}+\mathrm{H}_{2} \mathrm{O} \\
2 \mathrm{HCr}_{2} \mathrm{O}_{7}^{-} \rightleftarrows \mathrm{Cr}_{4} \mathrm{O}_{13}{ }^{--}+\mathrm{H}_{2} \mathrm{O} .
\end{gathered}
$$

Die Bildung dieser verschiedenen Chromationen ist von der Wasserstoffionenkonzentration sehr abhängig. Diese hochpolymerisierten Chromationen entstehen auch in Chromsäurelösungen in Anwesenheit genügend grosser Mengen einer fremden Säure, z.B. Schwefelsäure, wie bei der vorliegenden Untersuchung.

Nun braucht man für Oxydation von $\mathrm{Hu}-$ mus konzentrierte Chromschwefelsäurelösungen, welche dann für die Kolorimetrierung mit Wasser verdünnt werden müssen. Die grüne Farbenkomponente gibt sofort nach Verdünnen dieser Lö-

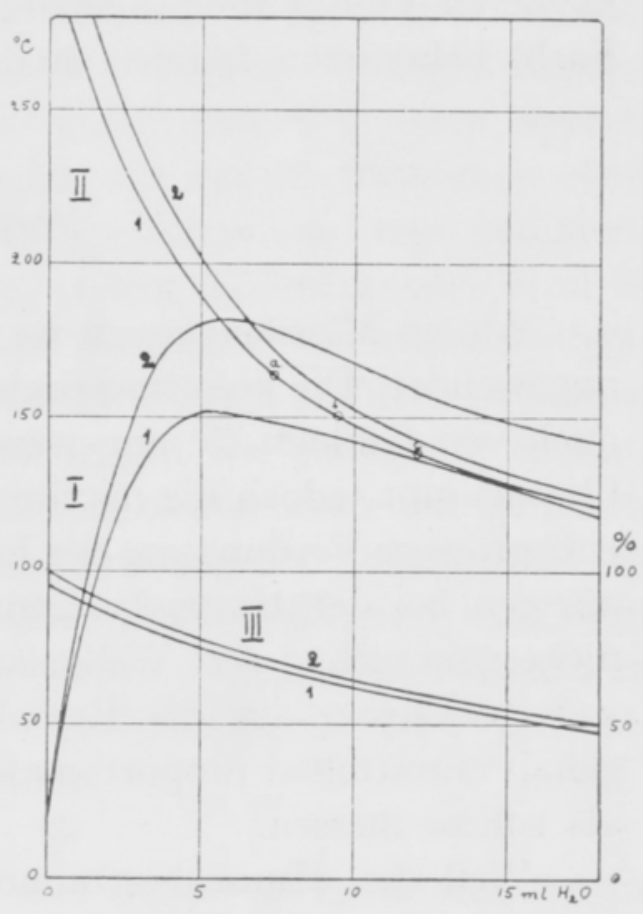

Fig 1. Die höchste theoretische Temperatur (y-Achse), die durch Hinzufügen verschieden grosser Wassermengen (x-Achse) bei Zimmertemperatur zu $10 \mathrm{ml} 96$ bzw. $100 \%$ Schwefelsäure (Kurven 1 bzw. 2) erreicht wird (Kurvenpaar I), nebst der Siedetemperatur (Kurvenpaar II) und den Konzentrationen in Gewichtsprozent (Kurvenpaar III) dieser Lösungen. Die Punkte a, b bzw. c zeigen die Siedetemperatur der bei der Methode SPRINGER \& KLEE, unserer Methode III (S. 206) bzw. der Methode WesterHOFF angewendeten Schwefelsäurelösungen. 
sungen, auf der Wellenlänge $620 \mathrm{~m} \mu$ gemessen, bedeutend grössere Extinktionswerte als einige Stunden später. Auch danach verbleicht diese grüne Farbenkomponente kontinuierlich, aber doch bedeutend langsammer. Z.B. eine Lösung, die 25 mg-Äqu Chromisalz und 25 mg-Äqu Chromat auf $250 \mathrm{ml} 1.45 \mathrm{n}$ Schwefelsäurelösung enthält, gab auf der obenerwähnten Wellenlänge, mittels einer $10 \mathrm{~mm}$ Kuvette gemessen, folgende Extinktionsablesungen: sofort nach dem Verdünnen 0.735, 2 Stunden später 0.550 und nach 24 Stunden 0.530. Die rote Farbenkomponente der Lösung wird dabei gleichzeitig immer dunkler.

Das schnelle Verbleichen der grünen Farbenkomponente sofort nach dem Verdünnen der Chromschwefelsäurelösung beruht wahrscheinlich auf der Depolymerisation der grossen Chromationen, während das später langsam fortschreitende Verbleichen von der Oxydation des dreiwertigen Chroms abhängig ist. Die Depolymerisation der grossen Chromationen geschieht auch schon bei einer bedeutend kleineren Verdünnung, aber da solche Lösungen in der Praxis all zu starke grüne Farbe bekommen, können sie deshalb nicht in Frage kommen.

\section{Vorschläge zu neven Methoden}

Als ein Messinstrument wurde das Spektrophotometer von Beckman, Modell B, angewendet. Die kolorimetrischen Messungen wurden auf der Wellenlänge $620 \mathrm{~m} \mu$, auch von Graham (5) angewendet, ausgeführt. Die grüne Farbe des dreiwertigen Chroms gibt jedoch die maximale Ablesung auf der Wellenlänge $590 \mathrm{~m} \mu$. Aber um eine grössere Verdünnung der Lösungen oder eine Anwendung von kleineren Bodenmengen bei der Humusbestimmung zu vermeiden, wurde diese Wellenlänge dabei nicht gebraucht. Auf Wellenlängen, die grösser als $590 \mathrm{~m} \mu$ sind, wirkt das sechswertige Chrom auf die Extinktion nicht ein, und man kann die dem Humusgehalt unmittelbar proportionale grüne Farbenkomponente des dreiwertigen Chroms als solche messen.

Weil die Humusbestimmung durch eine Oxydation in Chromschwefelsäure vorzugsweise auf die Mineralböden abgesehen ist, wurden für diese Untersuchung 20 Bodenproben grösstenteils solcher Art ausgewählt. Unter ihnen waren jedoch auch einige Humusbodenproben und ein Sphagnum fuscum Torfboden. Diese lufttrockenen Bodenproben wurden in einem Mörser fein zermahlen und durch ein $0.5 \mathrm{~mm}$ Sieb passiert. Dann wurde von den auf diese Weise vorbehandelten Bodenproben eine etwa $130 \mathrm{mg}$ Humus entsprechende Bodenmenge in $250 \mathrm{ml}$ Erlenmeyer-Kolben abgewogen. Diese Humusmenge ist für die vorliegende, verschiedene Verfahren vergleichende Untersuchung geeignet, weil ungefähr eine Hälfte oder $25 \mathrm{mg}$-Äqu des zugesetzten sechswertigen Chroms in dreiwertiges Chrom reduziert wird, welches in einer Lösung vom $250 \mathrm{ml}$ Volumen auf Wellenlänge $620 \mathrm{~m} \mu$ mittels einer $10 \mathrm{~mm}$ Kuvette gemessen, eine günstige Farbenintensität mit einer Extinktion gibt, die etwas grösser als 0.5 ist.

Die Vergleichslösungen wurden durch Oxydieren verschiedener Mengen Oxalsäure, anstatt des Bodens, in Chromschwefelsäure hergestellt. Wie auch LAUNER 
und Tomimatsu (6) gezeigt haben, wird die Oxalsäure in diesem Falle sehr leicht und quantitativ oxydiert. Durch eine Oxydation von $633.8 \mathrm{mg} \mathrm{C}_{2} \mathrm{O}_{4} \mathrm{H}_{2} \cdot 2 \mathrm{H}_{2} \mathrm{O}$ entsteht dabei 10 mg-Äqu Chromisalz. Die Reduktionsfähigkeit der Oxalsäure wurde auch durch die Methode Alten kontrolliert. Durch eine titrimetrische Bestimmung wurden beinahe theoretische mg-Äqu-Werte der unverbrauchten Mengen des sechswertigen Chroms erhalten, und die durch eine kolorimetrische Bestimmung des dabei gebildeten dreiwertigen Chroms erhaltenen Extinktionswerte zeigten einen fast geradlinigen Gang.

Nach zahlreichen Vorexperimenten wurden folgende drei kolorimetrischen $\mathrm{Hu}-$ musbestimmungsmethoden, die zur Zeit als die besten betrachtet werden können, entwickelt.

Methode I: Erhitzen auf dem siedenden Wasserbad. Von den feinzermahlenen Bodenproben werden in $250 \mathrm{ml}$ Erlenmeyer-Kolben die etwa $130 \mathrm{mg}$ Humus enthaltenden Bodenmengen gewogen. Für die Herstellung der Vergleichslösungen werden die in der Tabelle 5 erwähnten Mengen Oxalsäure genommen. Zu diesen werden $10 \mathrm{ml} 96 \%$ Schwefelsäure hinzugefügt und gut geschüttelt, danach bleiben sie etwa 10 Minuten lang oder so lange, bis diese Säure auch allen anderen in Frage kommenden Kolben der Versuchsreihe zugeführt worden ist, stehen. Weiter werden $5 \mathrm{ml} 10$ n Chromsäure (100 $\mathrm{g} \mathrm{CrO}_{3}$ in $300 \mathrm{ml}$ Lösung) jedem Kolben in derselben Reihenfolge zugesetzt, danach schüttelt man. Dann werden die Kolben auf dem siedenden Wasserbad eine Stunde lang (eine halbe Stunde ist so gut wie genügend) erhitzt. Nach der Abkühlung werden die konzentrierten Chromschwefelsäurelösungen mittels eines Masskolbens auf $250 \mathrm{ml}$ verdünnt. Um die Trübung der Lösungen zu beseitigen, lässt man sie über Nacht stehen, wobei ihre Farbe genügend verbleicht (s. S. 204) und so gut wie stabilisiert wird. Zuletzt werden diese klaren, verdünnten Lösungen kolorimetriert. Je nach Bedarf kann man die Trübung der Lösungen entweder durch Zentrifugieren oder Filtrieren entfernen.

Methode II: Ohne Wärmezufuhr von aussen. Bei dieser Methode werden die Oxydation des Humus und die weitere Behandlung auf dieselbe Weise wie bei der Methode I ausgeführt, nur mit dem Unterschied, dass, um die Reaktionswärme besser zu behalten, ein grösseres Volumen Chromschwefelsäure (20 ml 96 \% Schwefelsäure $+10 \mathrm{ml} 5 \mathrm{n}$ Chromsäure) angewendet wird. Die Temperatur steigt hierbei ungefähr bis $120^{\circ} \mathrm{C}$. Die Kolben werden nach der Säurezufuhr in ein effektives Isoliermaterial, wie z.B. bei dieser Untersuchung in Glaswolle, in der sie lange heiss aufbewahrt werden, für $1-1 \frac{1}{2}$ Stunden gelegt. Wie schon früher erwähnt, entsteht dabei die Wärmereaktion nur durch die Verdünnung der konzentrierten Schwefelsäure und die Verbrennung der organischen Substanz. Ohne Wärmeisolierung kühlen die heissen Lösungen bei Zimmertemperatur schnell ab, und die Oxydation des Humus wird somit unvollständig. In der Praxis ist es bei dieser Methode vorteilhaft, möglichst konzentrierte Schwefelsäure und zylinderförmige Kolben anzuwenden. Für die Wärmeaufbewahrung kann man eine spezielle Isolierkiste, die mit passenden Aushöhlungen für solche Kolben versehen ist, konstruieren. Bei 
Tabelle 3. Humusgehalt in Versuchsbodenproben nach verschiedenen Methoden.

\begin{tabular}{|c|c|c|c|c|c|c|c|c|c|}
\hline \multirow{3}{*}{\multicolumn{2}{|c|}{ Probe }} & \multirow[b]{3}{*}{ Bodenart } & \multirow{3}{*}{$\begin{array}{c}\text { Einwaage } \\
\mathrm{g}\end{array}$} & \multicolumn{6}{|c|}{$\%$ Humus von Trockensubstanz } \\
\hline & & & & \multicolumn{5}{|c|}{ Methoden } & \multirow{2}{*}{$\begin{array}{l}\text { Glüh- } \\
\text {-verlust }\end{array}$} \\
\hline & & & & Alten & $\begin{array}{l}\text { Wasser- } \\
\text { bad }\end{array}$ & $\begin{array}{l}\text { Eigener- } \\
\text { hitzung }\end{array}$ & \multicolumn{2}{|c|}{$\begin{array}{c}\text { III } \\
\text { Kochen }\end{array}$} & \\
\hline & & Sph. fuscum Torf & 0.2 & 84.9 & 86.0 & 84.4 & 86.0 & 86.0 & 95.1 \\
\hline $\mathrm{AC}$ & 165 & Braunmoos-Seggentorf & 0.2 & 78.7 & 78.2 & 78.4 & 75.1 & 77.3 & 73.0 \\
\hline $\mathrm{AE}$ & 248 & Seggentorf & 0.3 & 52.9 & 53.0 & 52.4 & 51.7 & 54.4 & 51.0 \\
\hline $\mathrm{J}$ & 602 & Anmooriger Sand & 0.7 & 27.4 & 27.5 & 26.3 & 27.4 & 26.3 & 24.6 \\
\hline $\mathrm{AE}$ & 249 & Anmooriger Lehm & 0.7 & 21.2 & 21.6 & 20.7 & 21.3 & 21.8 & 21.3 \\
\hline $\mathrm{AE}$ & 589 & Bröckelton & 1.0 & 16.6 & 16.6 & 16.5 & 16.4 & 16.9 & 18.9 \\
\hline $\mathrm{H}$ & 1382 & Humoser Sand & 1.5 & 11.6 & 11.4 & 10.8 & 11.0 & 11.3 & 10.8 \\
\hline $\mathrm{AE}$ & 251 & Sandiger Lehm & 1.3 & 11.0 & 10.9 & 10.7 & 11.0 & 11.2 & 13.5 \\
\hline $\mathrm{AG}$ & 1129 & Lehm & 1.5 & 9.76 & 9.73 & 9.46 & 9.73 & 9.74 & 10.8 \\
\hline G & 1208 & Sand & 1.5 & 8.06 & 8.48 & 8.42 & 7.88 & 8.47 & 9.48 \\
\hline $\mathrm{AG}$ & 505 & Lehm & 2.0 & 7.04 & 7.04 & 7.20 & 7.16 & 7.24 & 9.36 \\
\hline $\mathrm{AE}$ & 250 & Toniger Schluff & 2.0 & 6.78 & 6.68 & 6.66 & 6.78 & 6.84 & 8.90 \\
\hline $\mathrm{AE}$ & 522 & Toniger Schluff & 2.2 & 6.30 & 6.44 & 6.35 & 6.22 & 6.31 & 7.52 \\
\hline I & 364 & Feinsand & 2.5 & 5.70 & 5.76 & 5.61 & 5.82 & 5.81 & 6.32 \\
\hline $\mathrm{O}$ & 4121 & Sandiger Lehm & 3.0 & 5.54 & 5.39 & 5.53 & 5.72 & 5.68 & 9.22 \\
\hline $\mathrm{AE}$ & 253 & Sand & 3.0 & 4.64 & 4.70 & 4.58 & 4.63 & 4.79 & 5.91 \\
\hline I & 63 & Schluffiger Lehm & 3.0 & 4.62 & 4.64 & 4.52 & 4.38 & 4.67 & 5.98 \\
\hline $\mathrm{AG}$ & 611 & Feinsand & 3.0 & 4.19 & 4.11 & 3.97 & 4.16 & 4.16 & 5.30 \\
\hline $\mathrm{AE}$ & 252 & Sand & 4.0 & 3.29 & 3.26 & 3.19 & 3.25 & 3.35 & 4.19 \\
\hline \multirow[t]{4}{*}{$\mathrm{AG}$} & 1 & Schwerer Ton, Untergrund & 8.0 & 1.48 & 1.43 & 1.43 & 1.44 & 1.44 & 3.49 \\
\hline & & Summe & & 371.70 & 372.86 & 367.12 & 367.07 & 373.70 & 394.67 \\
\hline & & Verhältniszahl der Summe & & 100.0 & 100.2 & 98.8 & 98.8 & 100.5 & 106.1 \\
\hline & & $\begin{array}{l}\text { Mittelwert der Verhältnis- } \\
\text { zahlen }\end{array}$ & & 100.0 & 100.0 & 98,3 & 99.2 & 100.9 & 122.8 \\
\hline
\end{tabular}

dieser Untersuchung gebrauchte man jedoch $250 \mathrm{ml}$ Erlenmeyer-Kolben, welche dann in einer Pappschachtel mit Glaswolle isoliert wurden, was weniger effektiv ist.

Methode III: Kochen auf freier Flamme. Nach dieser Methode wird die Oxydation des Humus in $250 \mathrm{ml} \mathrm{Kjeldahl-Kolben} \mathrm{ausgeführt.} \mathrm{Auf} \mathrm{dieselbe} \mathrm{Weise} \mathrm{wie} \mathrm{bei}$ den vorigen Methoden werden auch hier Bodenproben bzw. Oxalsäure gewogen und diesen $10 \mathrm{ml} 96 \%$ Schwefelsäure zugesetzt. Danach werden $10 \mathrm{ml} 10 \mathrm{n}$ Chromsäure zugeführt, und die Mischung wird 20 Minuten unter dem Rückflusskühler — hier wurde ein $50 \mathrm{~cm}$ langes Glasrohr mit $5 \mathrm{~mm}$ Durchmesser angewendet - gekocht. Ohne Rückflusskühler verdunsten bedeutende Mengen Wasser, was eine Extrazersetzung des Chromats veranlasst.

Die Tabelle 3 zeigt den Humusgehalt der sowohl nach Alten als auch nach den in dieser Arbeit vorgeschlagenen Methoden analysierten Versuchsböden, in Prozent 
der Trockensubstanz berechnet. Ausserdem wurden auch die Summen dieser Humusprozentsätze für jede Methode gesondert und ihre Verhältniszahlen gerechnet, wobei die Summe nach Alten mit 100 angesetzt war. Wie man aus dieser Tabelle ersieht, erhält man durch die Methoden I und III ungefähr gleich grosse Werte wie durch die Methode Alten und durch die Methode II etwas geringere Werte.

Um die Einwirkung der Kochdauer auf die Ergebnisse zu untersuchen, wurden die Mischungen sowohl 10 als auch 30 Minuten auf freier Flamme gekocht. Wie die Tabelle 3 zeigt, ist es am besten, ein etwa 20 Minuten langes Kochen anzuwenden.

In die letzten Kolumne der Tabelle 3 sind auch die Glühverlustzahlen der Versuchsböden vergleichshalber aufgenommen worden. Bei dem Sphagnum fuscum Torfboden beruht die Differenz zwischen der Glühverlustzahl und anderen Werten wahrscheinlich darauf, dass der Kohlenstoffgehalt in der organischen Substanz des Torfes anders als im Bodenhumus ist. Die Ursache dessen, dass die Glühverlustzahlen bei den tonhaltigen Böden bedeutend grösser als die anderen Werte sind, liegt darin, dass auch das Kristallwasser des Tones beim Glühen ausgeht.

In der untersten Zeile der Tabelle 3 sieht man die Mittelwerte der Verhältniszahlen, die erst jede für sich in Prozent von den Alten-Werten berechnet worden waren. Sie sind auch von derselben Grösse wie die aus der Summen der Humusprozente berechneten Verhältniszahlen. Hier ist nur der Mittelwert der Glühverlustzahlen bedeutend grösser als die Verhältniszahl der Summe, was auf den hohen Verhältniszahlen der humusarmen Tonböden beruht.

\section{Bedentung der Einwaagegrösse}

Der folgende Versuch zeigt, in welchem Masse die bei den oben vorgeschlagenen Methoden erhaltenen Humusmengen von den gebrauchten Bodenmengen abhängig sind. Dazu wurden fünf schon in der Tabelle 3 erwähnte Bodenproben verschiedener Art angewendet. Von diesen wurden vier verschiedene Bodenmengen, die etwa 20,40,60 und $80 \%$ von der ganzen Menge des verbrauchten sechswertigen Chroms oxydiert werden konnten, für jede Methode separat abgewogen. Am Ende der Reaktion verbleiben demnach in den Chromschwefelsäurelösungen verschiedene Mengen des Chromats, was auf die Oxydationsfähigkeit dieses Systems natürlich einwirkt. Die Ergebnisse dieses Versuchs ersieht man aus der Tabelle 4. Bei jeder von diesen neuen Methoden, unabhängig von den dazu gebrauchten Bodenmengen; wurde ein so gut wie gleich grosser Humusgehalt gefunden. Die grösste Abweichung in dieser Beziehung hat der schwere Untergrundtonboden AG 1 bei der Methode Alten erwiesen. Dabei hat man um so mehr Humus erhalten, je weniger Boden für die Analyse genommen worden ist. Fast alle Methoden haben bei kleinsten Einwagen den grössten Humusgehalt aufgewiesen. Auch hier wurden Verhältniszahlen der Summen auf dieselbe Weise wie in der Tabelle 3 berechnet. In der Praxis ist es vorteilhaft, dass man bei diesen neuen Methoden verhältnismässig grosse Bodeneinwagen für die Analyse anwenden kann. 
Tabelle 4. Einwirkung der Einwagegrösse bei verschiedenen Methoden.

\begin{tabular}{|c|c|c|c|c|c|c|}
\hline \multirow{4}{*}{$\begin{array}{c}\text { Probe und } \\
\text { Bodenart }\end{array}$} & \multirow{4}{*}{$\begin{array}{c}\text { Einwage } \\
\mathrm{g}\end{array}$} & \multicolumn{5}{|c|}{$\%$ Humus von Trockensubstanz } \\
\hline & & \multirow{3}{*}{ Alten } & & \multicolumn{3}{|l|}{ Methoden } \\
\hline & & & I & \multirow{2}{*}{$\begin{array}{c}\text { II } \\
\text { Eigener- } \\
\text { hitzung }\end{array}$} & \multicolumn{2}{|c|}{ III Kochen } \\
\hline & & & Wasserbad & & $10 \mathrm{~min}$ & $30 \mathrm{~min}$ \\
\hline \multirow{4}{*}{$\begin{array}{l}\text { AE } 248 \\
\text { Seggentorf }\end{array}$} & 0.105 & 52.8 & 52.8 & 51.2 & 54.8 & 57.0 \\
\hline & 0.210 & 53.0 & 53.2 & 52.3 & 51.4 & 54.3 \\
\hline & 0.315 & 52.8 & 52.8 & 52.4 & 51.8 & 54.6 \\
\hline & 0.420 & 52.3 & 51.8 & 52.7 & 51.4 & 52.4 \\
\hline \multirow{4}{*}{$\begin{array}{l}\text { AE } 589 \\
\text { Bröckelton }\end{array}$} & 0.32 & 16.9 & 16.6 & 16.6 & 17.0 & 18.6 \\
\hline & 0.64 & 16.7 & 16.5 & 16.2 & 16.6 & 17.0 \\
\hline & 0.96 & 16.2 & 16.3 & 16.7 & 16.2 & 16.8 \\
\hline & 1.28 & 16.4 & 16.3 & 16.1 & 16.1 & 16.5 \\
\hline \multirow{4}{*}{$\begin{array}{l}\text { AE } 522 \\
\text { Toniger } \\
\text { Schluff }\end{array}$} & 0.8 & 6.68 & 6.64 & 6.64 & 6.48 & 6.48 \\
\hline & 1.6 & 6.36 & 6.56 & 6.36 & 6.24 & 6.42 \\
\hline & 2.4 & 6.22 & 6.33 & 6.35 & 6.16 & 6.29 \\
\hline & 3.2 & 6.26 & 6.32 & 6.34 & 6.10 & 6.33 \\
\hline \multirow{4}{*}{$\begin{array}{l}\text { AE } 253 \\
\text { Sand }\end{array}$} & 1.1 & 4.80 & 4.66 & 4.70 & 4.75 & 4.94 \\
\hline & 2.2 & 4.63 & 4.72 & 4.61 & 4.62 & 4.80 \\
\hline & 3.3 & 4.64 & 4.68 & 4.55 & 4.63 & 4.78 \\
\hline & 4.4 & 4.62 & 4.59 & 4.66 & 4.55 & 4.78 \\
\hline \multirow{4}{*}{$\begin{array}{l}\text { AG } 1 \\
\text { Schwerer Ton } \\
\text { Untergrund }\end{array}$} & 3.3 & 1.58 & 1.44 & 1.47 & 1.44 & 1.54 \\
\hline & 6.6 & 1.58 & 1.43 & 1.46 & 1.44 & 1.45 \\
\hline & 9.9 & 1.46 & 1.42 & 1.41 & 1.43 & 1.42 \\
\hline & 13.2 & 1.42 & 1.46 & 1.40 & 1.44 & 1.45 \\
\hline \multirow{2}{*}{\multicolumn{2}{|c|}{$\begin{array}{l}\text { Summe } \\
\text { Verhältniszahl }\end{array}$}} & 327.35 & 326.55 & 324.15 & 324.58 & 337.88 \\
\hline & & 100.0 & 99.8 & 99.1 & 99.2 & 103.1 \\
\hline
\end{tabular}

Tabelle 5. Durchschnittliche Extinktionswerte der Vergleichslösungen.

\begin{tabular}{|c|c|c|c|c|c|c|c|c|c|c|c|}
\hline \multirow{4}{*}{$\begin{array}{c}\mathrm{C}_{2} \mathrm{O}_{4} \mathrm{H}_{2} \cdot 2 \mathrm{H}_{2} \mathrm{O} \\
\mathrm{mg}\end{array}$} & \multirow{4}{*}{$\begin{array}{c}\mathrm{Cr}^{+++} \\
\mathrm{mg}-\ddot{\mathrm{Aqu}} \\
250 \mathrm{ml}\end{array}$} & \multicolumn{10}{|c|}{ Methoden } \\
\hline & & \multirow{2}{*}{\multicolumn{2}{|c|}{ Alten }} & \multirow{2}{*}{\multicolumn{2}{|c|}{$\begin{array}{c}\text { I } \\
\text { Wasserbad }\end{array}$}} & \multirow{2}{*}{\multicolumn{2}{|c|}{$\begin{array}{l}\text { II } \\
\text { Eigener- } \\
\text { hitzung }\end{array}$}} & \multicolumn{4}{|c|}{ III Kochen } \\
\hline & & & & & & & & & $10 \mathrm{~min}$ & & $30 \mathrm{~min}$ \\
\hline & & * & Extinkt. & * & Extinkt. & * & Extinkt. & * & Extinkt. & * & Extinkt. \\
\hline 633.8 & 10 & 4 & 0.219 & 3 & 0.214 & 1 & 0.212 & 1 & 0.221 & 1 & 0.227 \\
\hline 1267.5 & 20 & 2 & 0.426 & 1 & 0.410 & 1 & 0.422 & 1 & 0.421 & 1 & 0.425 \\
\hline 1584.4 & 25 & 5 & 0.531 & 8 & 0.525 & 3 & 0.528 & 3 & 0.521 & 2 & 0.521 \\
\hline 1901.3 & 30 & 4 & 0.628 & 3 & 0.622 & 4 & 0.631 & 3 & 0.621 & 3 & 0.624 \\
\hline 2535.0 & 40 & 3 & 0.838 & 4 & 0.834 & 1 & 0.835 & 1 & 0.825 & 1 & 0.830 \\
\hline
\end{tabular}

* Anzahl Beobachtungen. 
Zum Schluss sieht man in der Tabelle 5 die durchschnittlichen Extinktionswerte der Vergleichslösungen, die man zu verschiedener Zeit nach den obenerwähnten Methoden durch Oxydation der Oxalsäure hergestellt und auf Wellenlänge $620 \mathrm{~m} \mu$ mittels einer $10 \mathrm{~mm}$ Kuvette kolorimetriert hat. Die Extinktionswerte der Vergleichslösungen unterscheiden sich bei diesen verschiedenen Methoden nicht besonders von einander, weshalb angenommen werden kann, dass in allen diesen Fällen die Zersetzung des Chromats so gut wie gleich gross ist. In der Annahme, dass bei der Methode Alten eine nur sehr kleine Extrazersetzung des Chromats eintritt, wird sie auch bei diesen neuen Methoden wahrscheinlich unbedeutend sein. Man darf jedenfalls hoffen, dass die Zersetzung des Chromats in Vergleichslösungen wie auch in Versuchslösungen bei diesen Methoden auf dieselbe Weise geschieht und dass eine eventuelle Ungenauigkeit beim Berechnen der Ergebnisse durch den Vergleich dieser Lösungen verschwinden wird. Natürlich wird aber in Anwesenheit des Bodens, der als eine Art Katalysator wirken kann, der Gang der Reaktion vielleicht ein wenig abweichend sein.

\section{Zusammenfassung}

In der vorliegenden Untersuchung wurden einige wichtige bisherige Verfahrensweisen, den Humus durch die Oxydation in Chromschwefelsäurelösungen zu bestimmen, verglichen und kritisiert.

Auch der Mechanismus der Oxydation des Humus wurde u.a. unter Anwendung des Oxydations-Reduktionspotentials, der Lösungswärme der konzentrierten Schwefelsäure und der Siedetemperatur der Schwefelsäurelösungen verschiedener Konzentration theoretisch analysiert.

Als Oxydationsmittel wurde Chromtrioxyd aus dem Grunde, weil es im Wasser viel löslicher als Kaliumdichromat ist, zur Anwendung vorgeschlagen.

Bei dieser Untersuchung wurden drei neue Methoden für kolorimetrische $\mathrm{Hu}-$ musbestimmung entwickelt: 1. Durch Erhitzen im siedenden Wasserbad, 2. ohne Wärmezufuhr von aussen und 3. durch Kochen auf freier Flamme.

Dazu wurden 20 Bodenproben von sehr verschiedenem Humusgehalt sowohl durch diese neuen Methoden als auch durch die zu einer kolorimetrischen veränderten Methode Alten analysiert und die Anwendung dieser neuen Verfahrensweise durch Vergleich der Ergebnisse kritisiert.

Für die Herstellung der Vergleichslösungen wurde Oxalsäure angewendet, die sich in Chromschwefelsäurelösungen durch die obenerwähnten Methoden leicht oxydiert und die stöchiometrischen Mengen des Chroms reduziert.

Ich gestatte mir an Professor Dr. Martti Salonen und Doktor Armo Tern̈svUORI meinen verbindlichsten Dank für allseitige Unterstützung, wertvolle Ratschläge und mehrere Besprechungen bei dieser Arbeit zu richten. 


\section{LITERATUR}

(1) Aarnio, B. 1931. Maaperäoppi, p. 1-194.

(2) Alten, F., Wandrowsky, B. \& Knippenberg, E. 1935. Beitrag zur Humusbestimmung. Ergebnisse der Agrikulturchemie, 4, p. 61-69.

(3) Bondorff, K. A. 1946. Om Humusbestemmelse i Jord. Tidsskrift for Planteavl, 50, p. 138 - 149.

(4) Carolan, R. 1948. Modification of Graham's method for determining soil organic matter by colorimetric analysis. Soil Sci., 66, p. $241-247$.

(5) Graham, E. R. 1948. Determination of soil organic matter by means of a photoelectric colorimeter. Ibid., 65 , p. $181-183$.

(6) Launer, H. F. \& Tomimatsu, Y. 1953. Rapid accurate determination of carbo hydrates and other substances with the dichromate heat-of-dilution method. Anal. Chem., 25, p. 1767-1769.

(7) OKNin, I. V. 1949. Oxidative properties of solutions of sulfuric acid. Zhur. Priklad. Khim. J. Applied Chem., 22, p. 1143-1149. Ref.: Chemical Abstracts 1950, 2831a.

(8) Rauterberg, E. \& Sandhoff, H. 1951. Eine Schnellmethode zur Bestimmung der Humusstoffe in Böden. Z. Pfl. Ernähr. Düng. Bodenkunde, 55 (100), p. 15-23.

(9) Salonen, M. 1941. Fosforin esiintymismuodoista Suomen maalajeissa. Zusammenfassung: Über die Formen des Vorkommens von Phosphor in den Bodenarten Finnlands. Acta Agralia Fenn., 48, p. 1-124.

(10) Springer, U. 1948. Möglichkeiten zur schnellen Humusbestimmung im Boden. Z. Pfl. Ernähr. Düng. Bodenkunde, 40(85), p. 166-169.

(11) Springer, U. \& Klee, J. 1954. Prüfung der Leistungsfähigkeit von einigen wichtigeren Verfahren zur Bestimmung des Kohlenstoffs mittels Chromschwefelsäure sowie Vorschlag einer neuen Schnellmethode. Ibid., 64(109), p. 1-26.

(12) Tuorila, P., Tainio, A. \& Teräsvuori, A. 1939. Suomen viljelysmaiden kalkitustarpeesta. Referat: Über den Kalkdüngungsbedarf der finnischen Böden. Valt. maatalousk. julk., 104, p. $1-529$.

(13) Vuorinen, J. 1946. Maaperän humuksen määrittämisestä. Summary: Determination of humus in soil. Maataloust. Aikak., 18, p. 11-24.

(14) WalkLey, A. 1947. A critical examination of a rapid method for determining organic carbon in soils - Effect of variations in digestion conditions and of inorganic soil constituents. Soil Sci., 63, p. 251-264.

(15) Westerhoff, H. 1952. Beitrag zur kolorimetrischen Humusbestimmung im Boden. Z. Pfl. Ernähr. Düng. Bodenkunde, 56(101), p. $49-53$.

\section{SEL OS T U S:}

\section{LISIÄ MAAN HUMUKSEN KOLORIMETRISEEN MÄÄRITTÄMISEEN}

\section{EUGEN BARKOFF}

\section{Maatalouskoelaitos, maanviljelyskemian ja -fysiikan osasto, Tikkurila}

Esilläolevassa tutkimuksessa on vertailtu ja arvosteltu eräitä tärkeimpiä tähänastisia humuksen määrittämistapoja kromirikkihappoliuoksilla. Myös humuksen hapettamisen mekanismia on analysoitu teoreettisesti mm. hapetus-pelkistyspotentiaalia, väkevän rikkihapon laimentamislämpöä ja eri väkevien rikkihappoliuosten kiehumislämpötiloja hyväksi käyttäen. Hapettimena on ehdotettu käytettäväksi kaliumdikromaatin sijasta kromitrioksidia tämän suuremman vesiliukoisuuden takia.

Tutkimuksen perusteella on kehitetty kolme uutta muunnelmaa humuksen kolorimetrisille määrittämistavoille: 1. lämmittämällä vesihauteella, 2. ilman ulkopuolista lämmittämistä ja 3 . keittämällä vapaalla liekillä. Humusmääritykset tehtiin 20:stä hyvin erilaista humuspitoisuutta edustavasta maanäytteestä sekä näillä uusilla tavoilla että kolorimetriseksi muutetulla ALtENin tavalla ja tuloksia vertaamalla arvosteltiin yllämainittujen uusien tapojen käyttökelpoisuutta. Vertailuliuosten valmistamiseen on käytetty oksaalihappoa, joka hapettuu helposti yllämainittujen menetelmien mukaan kromihappoliuoksissa ja pelkistää stökiometriset kromimäärät.

\section{Yliopiston}

